\title{
Long Term Evaluation of Intensive Insulin Therapy in Patients with Type 2 Diabetes Mellitus
}

\author{
Valdez MNR ${ }^{1}$ and Mercado-Asis LB ${ }^{2 *}$ \\ ${ }^{1}$ Section of Endocrinology, Diabetes and Metabolism, University of Santo Tomas Hospital, Manila, Philippines \\ ${ }^{2}$ Faculty of Medicine and Surgery, University of Santo Tomas, Manila, Philippines
}

"Correspondence: Leilani B Mercado-Asis, Faculty of Medicine and Surgery, University of Santo Tomas, Manila, Philippines

Received on 14 September 2020; Accepted on 01 February 2021; Published on 06 February 2021

Copyright () 2020 Valdez MNR, et al. This is an open access article and is distributed under the Creative Commons Attribution License, which permits unrestricted use, distribution, and reproduction in any medium, provided the original work is properly cited.

\begin{abstract}
Background: This study aimed to determine long-term effect of intensive insulin therapy on prevention, progression, and development of chronic diabetes complications, both micro and macrovascular events. This study also aimed to evaluate long-term sustainability of glycemic control of patients on intensive insulin treatment.

Methods: A retrospective review of adult type 2 diabetes mellitus (T2DM) patients on intensive insulin therapy for $\geq 7$ years. Demographic data, co-morbidities, body mass index (BMI), hemoglobin Alc (HbAlc), hospitalization were collated. Majority received intensive insulin therapy with combination of premixed 70/30 given two times a day and fast short acting analogue given premeal three times a day, with the addition of glargine or degludec once a day in some.

Results: Among 76 patients, $62 \%$ were males and $38 \%$ were females. Mean age at diagnosis and last visit were 53 and 65 years, respectively. At time of diagnosis, patient had the following co-morbidities: hypertension (32\%), dyslipidemia (13\%), non-dialyzable chronic kidney disease (CKD) (4\%), thyroid disease (1\%), pulmonary tuberculosis (1\%). In terms of long-term complications, event rates during follow up period are as follows: 0.001 per person-year for acute coronary event; 0.002 per person-year for CKD needing dialysis, 0.009 per person-year for cerebrovascular accident. There were no blindness and amputation observed. There is a statistical difference between HbAlc levels at time of diagnosis $(8.53 \pm 1.86)$ and last follow up $(7.83 \pm 1.71)(P=0.00)$. After a median follow up of 12 years $(7-22$ years $)$, glycemic control was sustained with an HbAlc of $\leq 7 \%$ and $\leq 8 \%$ in $32 \%$ and $45 \%$ of patients, respectively.

Conclusion: With intensive insulin therapy, micro and macrovascular complications can be prevented significantly. Long-term sustainability of glycemic control was also achieved.
\end{abstract}

Keywords: type 2 diabetes mellitus, intensive insulin therapy, diabetes complications, glycemic control, retrospective study 
Abbreviations: T2DM: type 2 diabetes mellitus; BMI: body mass index; HbA1c: hemoglobin A1c; CKD: chronic kidney disease; NHANES: National Health and Nutrition Examination Survey; IRB: Institutional Review Board; NPH: neutral protamine Hagedorn; DPP-4: dipeptidyl peptidase-4; ACS: acute coronary syndrome

\section{Introduction}

Diabetes as a chronic disease has an upward trend of prevalence and incidence worldwide exerting a major impact in third-world countries, predominantly in the Philippines. There are 3.2 million cases of type 2 diabetes mellitus (T2DM) in the Philippines with a $5.9 \%$ prevalence rate in adults between the ages of 20 and 79 years as projected by the International Diabetes Federation in 2014. The highest prevalence rate was found among the richest in the wealth index, those living in urban areas, and those in the 60-69-year age group in both sexes [1].

Over time, diabetes can lead to serious complications classified as microvascular or macrovascular. Data from the 1999-2004 National Health and Nutrition Examination Survey (NHANES) pointed out that the prevalence of microvascular complications such as chronic kidney disease (CKD), foot problems, and eye damage are much higher than the prevalence of macrovascular complications including cardiovascular disease, stroke, and peripheral vascular disease [2].

T2DM has become a developing cause of morbidity and mortality primarily through cardiovascular disease and other chronic complications. These chronic complications being the major outcome of T2DM advancement reduce quality of life and contribute to health care cost [3].

Cardiovascular disease accounts for up to $65 \%$ of all deaths in people with diabetes. Risk factors such as hypertension, hypercholesterolemia, and smoking, lead to poorer outcomes with increased mortality rates [2]. Peripheral arterial disease as a significant risk factor for lower-extremity amputation is increased by age, duration of diabetes and presence of neuropathy. Diabetic retinopathy results in more than 10,000 new cases of blindness per year and is well linked to prolonged hyperglycemia.

Progressive deterioration of beta cell function and insulin resistance characterize T2DM regardless of lifestyle and current pharmacological interventions $[4,5]$. With its rapidly growing prevalence, it remains the principal cause of blindness, end-stage renal disease, lower-limb amputation and cardiovascular disease [6]. It has been a wellrecognized risk factor for correlation between measures of glycemic control and both cardiovascular outcomes and microvascular disease [7].

Even with the advent of newer therapies for treatment of diabetes, it is still linked to frequent incidence of microvascular and macrovascular complications [6]. High cardiovascular risk is a common problem associated with increased morbidity and mortality. Thus, management of risk factors for CVD such as hypertension, obesity, and dyslipidemia is a significant component in dealing with diabetes [8]. Randomized controlled trials have intended to evaluate whether a more intensive control of glucose lowers long-term clinical events compared to standard treatment $[9,10]$. Unluckily, these trials have failed to show consistent beneficial effects on cardiovascular events compared to microvascular outcomes [7].

Insulin therapy is most commonly introduced late in the course of the disease when oral anti-diabetics can no longer sustain glycemic control [11]. Unlike therapies that stimulate endogenous insulin secretion and requiring greater beta cell response, insulin therapy can provide some beta cell rest giving an opportunity to slow and possibly reverse betacell deterioration [12]. Moreover, it has been recently proposed that early insulin implementation in T2DM may lessen cardiovascular risk $[6,11]$. Intensive insulin therapies such as multiple daily insulin injections that target glycemic control and induce maintained euglycemia could enhance beta cell function resulting to longer remissions. However, it is still unclear which type of early intensive therapy is more advantageous and whether this effect is secondary to insulin therapy itself or to good glycemic control from glucotoxicity eradication [12]. To address such uncertainties, 
the present study aims to determine whether intensive insulin treatment in patients with T2DM will result to a reduction of chronic complications and further improve glycemic control.

\section{Materials and Methods}

\section{Study design and patients}

Medical charts of patients diagnosed with T2DM and on intensive insulin therapy for 7 years or more from an endocrine specialty clinic were reviewed retrospectively. The Institutional Review Board (IRB) of University of Santo Tomas Hospital approved this study. Diagnosis of T2DM was based on elevated hemoglobin A1c (HbA1c) level with the following HbA1c goals: $7 \%$ for patients aged 60 years and below, 7-8\% for patients aged 61 years and above.

Intensification of insulin therapy was initiated for patients not reaching their HbA1c targets despite 2 or more oral anti-diabetic drugs using the following insulin combinations: premixed NPH/rapid-acting (70/30) and rapid insulin; basal and rapid insulin; NPH and regular insulin; and premixed NPH/rapid-acting (70/30), basal with rapid insulin.

Chronic complications of diabetes were routinely checked on each follow up visit. In terms of cardiovascular and cerebrovascular diseases, clinical signs and symptoms of possible acute coronary event, heart failure, arrhythmia or stroke, with body mass index (BMI) and blood pressure readings are regularly assessed during clinic visit. Cholesterol levels are checked at least every 6 months to 1 year and more often if not on target. Skin changes, calluses, and foot ulcers, which may predispose patients to diabetic foot complications are also regularly examined at each visit. Annual dilated eye exams are advised to screen for diabetic retinopathy. Creatinine levels as well as presence of albuminuria are monitored at least every 3-6 months to evaluate for diabetic kidney disease.

Diagnosed T2DM patients were characterized based on demographics, BMI and presence of co-morbidities such as hypertension, dyslipidemia, non-dialyzable CKD, thyroid disease, and pulmonary disease (Table 1).

Long-term evaluation of HbA1c levels as well as occurrence of chronic complications reported within the follow up period were also documented (Table 2).

\section{Statistical analysis}

The statistical analysis was performed using the Statistical Package for the Social Sciences (SPSS). Results were reported as mean or percentage for individual characteristics. Student's t-test was used to compare the differences between the mean of the parameters. Differences were considered statistically significant at a level of $\mathrm{P}<0.05$.

\section{Results}

\section{Baseline characteristics}

The baseline characteristics of the 76 patients included in the study are summarized in the table (Table 1 ). Among 76 patients, $47(62 \%)$ were females and $29(38 \%)$ were males. Mean age at diagnosis and last visit were 53 and 65 years respectively. Patients had mean BMI of 26.46 at time of diagnosis and 28.31 at last visit. At time of diagnosis, patient had the following co-morbidities: hypertension (32\%), dyslipidemia (13\%), non-dialyzable CKD (4\%), thyroid disease $(1 \%)$, pulmonary tuberculosis $(1 \%)$.

\section{Long-term course on intensive insulin therapy}

In terms of long-term complications, event rates during follow up period are as follows: 0.001 per person-year for acute coronary event; 0.002 per person-year for CKD needing dialysis, 0.009 per person-year for cerebrovascular accident. There were no blindness and amputation observed during the course of follow up. There is a statistical difference between HbA1c levels at time of diagnosis $(8.53 \pm 1.86)$ and last follow up $(7.83 \pm 1.71)(\mathrm{P}=0.00)$. After a median follow up of 12 years (7-22 years), glycemic control was sustained with an HbA1c of $\leq 7 \%$ and $\leq 8 \%$ in $32 \%$ and $45 \%$ of patients, respectively (Table 2 ). 


\begin{tabular}{|l|l|}
\hline Variable & Value \\
\hline Age (years) & $53 \pm 11$ \\
\hline At diagnosis & $65 \pm 11$ \\
\hline At last visit & 62 \\
\hline Sex & 38 \\
\hline Female & \\
\hline Male & $26.46 \pm 4.14$ \\
\hline BMI (kg/m ${ }^{2}$ ) & $28.31 \pm 4.67$ \\
\hline At diagnosis & 32 \\
\hline At last visit & 13 \\
\hline Co-morbidities & 4 \\
\hline Hypertension & 1 \\
\hline Dyslipidemia & 1 \\
\hline Chronic kidney disease, non-dialyzable & \\
\hline Thyroid disease &
\end{tabular}

Table 1: Characteristics of type 2 diabetes mellitus patients on intensive insulin therapy. Values are expressed as mean \pm SD or number (\%); BMI: body mass index.

\begin{tabular}{|l|l|l|}
\hline Variable & Value & P value \\
\hline HbA1c & $8.53 \pm 1.86$ & 0.00 \\
\hline At diagnosis & $7.83 \pm 1.71$ & 0.00 \\
\hline At last visit & 32 & \\
\hline$\leq 7 \%$ & 45 & \\
\hline$\leq 8 \%$ & \multicolumn{2}{|l|}{} \\
\hline Microvascular complications & 0.002 & \\
\hline CKD needing dialysis & 0 & \\
\hline Blindness & \multicolumn{2}{|l|}{} \\
\hline Macrovascular complications & \multicolumn{5}{|l|}{} \\
\hline Acute myocardial infarction & 0.001 & \\
\hline Cerebrovascular accident & 0.009 & \\
\hline Amputation & 0 & \\
\hline
\end{tabular}

Table 2: Long-term course of type 2 diabetes mellitus patients on intensive insulin therapy. Values are expressed as mean \pm SD or rate (events per person-year); CKD: chronic kidney disease.

\section{Discussion}

In this retrospective analytical study of patients with T2DM, long-term macrovascular complications such as acute coronary event and cerebrovascular accident occurred less frequently with intensive insulin therapy. Amputation was not observed during the course of follow up. Microvascular complications such as CKD needing dialysis were noted only in a small percentage of patients with no experienced blindness. These clinical benefits were associated with a significant decrease in $\mathrm{HbAlc}$ from the time of diagnosis. Moreover, there were no reported major hypoglycemic events documented by Whipple's triad: symptoms of hypoglycemia, low plasma glucose concentration usually of $\leq 70$ $\mathrm{mg} / \mathrm{dL}$, and relief of symptoms after plasma glucose concentration is raised.

Several reasons why clinicians refrain from starting insulin or intensifying insulin treatment include the risk for hypoglycemia, weight gain and patient's anxiety over insulin use. In a study by Lopez et al. [13], a stepwise insulin combinations treatment algorithm was used as a guide for insulin intensification with the aim of HbA1c reduction taking into consideration hypoglycemic events and influence on weight gain. This treatment algorithm which includes oral regimen used singly or in combination (metformin, sulfonylurea, thiazolidinedione, dipeptidyl peptidase-4 (DPP4) inhibitor; regimen A (basal insulin + oral regimen); regimen B (basal insulin + pre-meal bolus three times daily \pm oral regimen except sulfonylurea); regimen $\mathrm{C}$ (premixed aspart 70/30 or premixed lispro 75/25 three times daily before meals, or premixed aspart 70/30 or premixed lispro 75/25 pre-breakfast and pre-dinner, + pre-lunch lispro or aspart bolus, \pm oral regimen); regimen $\mathrm{D}$ (premixed human insulin 70/30 twice daily + pre-meal bolus of aspart or lispro, \pm 
oral regimen); and regimen $\mathrm{E}$ (regimen D + basal insulin) may actually help physicians even non-specialists to adhere to early insulinization.

It has been well established that blood glucose elevation during acute coronary syndrome (ACS) is associated with increased morbidity and mortality. Pro-thrombotic and pro-inflammatory effects of hyperglycemia are linked to endothelial dysfunction. In a study by Marfella et al. [14] on glucose regulation in myocardial infarction, glycemic control can lessen remodeling and apoptosis by reducing oxidative stress and inflammation. Also, one study has showed that intensive insulin therapy can increase fibrinolytic process hence beneficial for patients with hyperglycemia at risk of ACS as well as ischemic stroke [14]. Present findings may additionally support this conclusion implying one rationale to the use of intensive insulin therapy.

Another dreaded macrovascular complication of uncontrolled hyperglycemia is the risk of amputation. Leukocyte dysfunction, cytokine dysregulation, neuropathy and vasculopathy have significant roles in impaired wound healing of diabetic foot ulcers [15]. In our study, no minor or major amputation was reported during the follow-up period even after hospitalization for foot ulcers. This result of our study indicates that intensive insulin therapy accelerates wound healing in patients with diabetes. This can be supported by the study of Vatankhah et al. [15], which demonstrated positive association of systemic insulin treatment with higher rates of complete wound healing.

Furthermore, we have showed that only 2 patients had CKD needing renal replacement therapy during the median follow-up of 12 years. Also, there was no report of blindness among the included patients. These findings establish that intensive control of blood glucose using multiple insulin injection therapy can efficiently delay development and worsening of diabetic nephropathy and retinopathy. A randomized prospective study using intensive insulin therapy confirms this outcome showing a delay in onset and progression of microvascular complications in a set of Japanese patients with T2DM [16].

In ensuring glycemic control, an HbA1c level should be regularly taken in addition to blood glucose monitoring. Abrahamson et al. [17] have proposed that if HbA1c and glucose values are beyond the individualized target level, bolus insulin should be added to basal insulin hence intensifying insulin replacement regimen. Of note, analysis of long-term glycemic control in this retrospective study showed a significant difference in $\mathrm{HbA} 1 \mathrm{c}(\mathrm{P}=0.00)$ from the time of diagnosis $(8.53 \pm 1.86)$ and last visit $(7.83 \pm 1.71)$. Also, a high rate of patients reached their target HbA1c goals ( $\leq 7 \%$ and $\leq 8 \%$ in $32 \%$ and $45 \%$ of patients respectively) within 12 years of median follow up. This sustained glycemic control of patients on intensive insulin therapy proves its positive effects on the delay in onset or progression of both micro and macrovascular complications in this set of patients.

Moreover, greater $\mathrm{HbA1c}$ reduction was seen in patients specifically on premixed insulin and lispro compared to premixed insulin alone in the study of Laplano et al [18]. This is attributed to the combination regimen's ability to mimic physiologic insulin response. In response to glucose, premixed NPH-regular insulin corresponds to release of newly formed insulin (Phase II) while lispro insulin matches with the rapid release of pre-formed insulin (Phase I).

Weight gain is a frequent side effect of insulin treatment. The more intensive the therapy such as the use of multiple daily insulin injections, the more weight gain is observed. In the present study, an increase from baseline body weight of $1.85 \pm 0.53 \mathrm{~kg}$ was observed during treatment with intensified insulin regimen.

Following insulin intensification, hypoglycemia is of major concern. An interesting result in this review is that there was no hypoglycemic event reported by patients during the follow up period. This can be explained by the provided proper patient education regarding automatic snacking and recognition of signs and symptoms of an impending hypoglycemic episode. This is supported by the study of Lorenzo et al. [19], which demonstrated that inclusion of three snacks taken automatically $2 \mathrm{~h}$ after each meal has decreased hypoglycemic events among T2DM patients on intensive insulin therapy.

\section{Conclusion}

In summary, the use of intensive insulin therapy showed that micro and macrovascular complications could be prevented significantly. Long-term sustainability of glycemic control was also achieved as evidenced by the significant reduction of $\mathrm{HbA} 1 \mathrm{c}$ to target level over a mean period of 12 years. 


\section{Limitations}

We acknowledge that this study was conducted in a single center and that involvement of multiple centers will be more appropriate to detect differences in individual primary outcomes in terms of micro and macrovascular complications related to sustained glycemic control.

\section{Conflicts of Interest}

No conflict of interest relevant to this article was reported.

\section{References}

1. Tan G. Diabetes care in the Philippines. Ann Glob Health. 2015;81(6):863-69.

2. Deshpande A, Harris-Hayes M, Schootman M. Epidemiology of diabetes and diabetes-related complications. Phys Ther. 2008;88(11):1254-264.

3. Liu Z, Fu C, Wang W, et al. Prevalence of chronic complications of type 2 diabetes mellitus in outpatients a cross-sectional hospital based survey in urban China. Health Qual Life Outcomes. 2010;8:62.

4. Mashitisho M, Mashitisho B. Early insulin therapy in patient with type 2 diabetes mellitus. J Endocrinol Metab Diabetes South Africa. 2016;21(1):13-15.

5. Weng J, Li Y, Xu W, et al. Effect of intensive insulin therapy on Beta-cell function and glycaemic control in patients with newly diagnosed type 2 diabetes: a multicentre randomised parallel-group trial. Lancet. 2008;371(9626):1753-760.

6. Chiasson J. Early Insulin Use in Type 2 Diabetes. Diabetes Care. 2009;32(Suppl 2):S270-S274.

7. Ray K, Seshasai S, Wijesuriya S, et al. Effect of intensive control of glucose on cardiovascular outcomes and death in patients with diabetes mellitus: a meta-analysis of randomised controlled trials. Lancet. 2009;373:1765-772.

8. Dailey G, Wang E. A Review of Cardiovascular Outcomes in the Treatment of People with Type 2 Diabetes. Diabetes Ther. 2014;5(2):385-402.

9. Mazzone T. Intensive Glucose Lowering and Cardiovascular Disease Prevention in Diabetes: Reconciling the Recent Clinical Trial Data. Circulation. 2010;122(21):2201-211.

10. Plank J, Kohler G, Rakovac I, et al. Long-term evaluation of a structured outpatient education programme for intensified insulin therapy in patients with Type 1 diabetes: a 12-year follow-up. Diabetologia. 2004;47(8):1370-375.

11. Retnakaran R, Drucker D. Intensive insulin therapy in newly diagnosed type 2 diabetes. Lancet. 2008;371(9626):1725-726.

12. Weng J, Retnakaran R, Ariachery A, et al. Short-term intensive insulin therapy at diagnosis in type 2 diabetes: plan for filling the gaps. Diabetes Metab Res Rev. 2015;31(6):537-44.

13. Lopez A, Mendoza E, Valdez V, et al. Treatment Outcomes with the Use of a Stepwise Insulin Combinations Algorithm Among Type 2 Diabetic Patients. Philippine Journal of Internal Medicine. 2016;54(2)1-7.

14. Bouida W, Beltaief K, Msolli M, et al. One-Year Outcome of Intensive Insulin Therapy Combined to Glucose-Insulin-Potassium in Acute Coronary Syndrome: A Randomized Controlled Study. J Am Heart Assoc. 2017;6(11):e006674. 
15. Vatankhah N, Jahangiri Y, Landry G, et al. Effect of systemic insulin treatment on diabetic wound healing. Wound Repair Regen. 2017;25(2):288-91.

16. Ohkubo $\mathrm{Y}$, Kishikawa H, Araki E, et al. Intensive insulin therapy prevents the progression of diabetic microvascular complications in Japanese patients with non-insulin-dependent diabetes mellitus: a randomized prospective 6-year study. Diabetes Res Clin Pract. 1995;28(2):103-117.

17. Abrahamson M, Peters A. Intensification of insulin therapy in patients with type 2 diabetes mellitus: An algorithm for basal-bolus therapy. Ann Med. 2012;14(8):836-46.

18. Laplano N, Quiambao D, Uy J, et al. Combination Insulin Therapy with Pre-Mixed Intermediate/RapidActing Insulin (70/30) and Insulin Lispro Mimicking Physiologic Insulin Secretion: Optimal Glycemic Treatment with Sustainability of Control in Type 2 Diabetic Patients. Philippine Journal of Internal Medicine. 2009;47:93-97.

19. Lorenzo Z, Asis L. Automatic snacking prevents hypoglycemia among outpatient type 2 diabetic patients on intensive insulin therapy. Philippine Journal of Internal Medicine. 2011;49(2)1-7. 